\title{
Design and validation of small interfering RNA on respiratory syncytial virus M2-2 gene: A potential approach in RNA interference on viral replication
}

\begin{abstract}
Human respiratory syncytial virus (RSV) is the leading cause of severe lower respiratory tract infection in infants and young children globally and is a significant pathogen of the elderly and immunocompromised. The M2-2 protein of respiratory syncytial virus (RSV) is particularly important in regulation of viral RNA transcription and replication that could be a potential anti-viral candidate against RSV infection. In this study, we designed and validated siRNAs that specifically target the RSV M2-2 gene. Four siRNAs targeting different regions of the M2-2 gene were designed using web tool. In-vitro evaluation of silencing effect was performed by using RSV infected Vero cell line. Viral M2-2 linked GFP recombinant plasmid was co-transfected with non-targeted siRNA, Pooled siRNA, siRNA 1, siRNA 2, siRNA 3 and siRNA 4 using synthetic cationic polymer. The silencing effect of M2-2 gene at the protein level was measured both qualitatively and quantitatively by using fluorescence microscopy and flow cytometry. Meanwhile, the silencing effect at the mRNA level was assessed by using RT-qPCR. This study showed that all four designed siRNAs can effectively and efficiently silence M2-2 gene. siRNA 2 showed the highest (98\%) silencing effect on protein level and siRNA 4 with $83.1 \%$ at the mRNA level. The viral assay showed no significant cytopathic effects observed after 6 days post-infection with siRNAs. In conclusion, this study showed the effectiveness of siRNA in silencing M2-2 gene both at the protein and mRNA level which could potentially be used as a novel therapeutic agent in the treatment of RSV infection. However, further study is warranted to investigate the silencing effect of M2-2 protein and inhibition of RSV infection.
\end{abstract}

Keyword: Antiviral; Gene silencing; Respiratory syncytial virus; RNA interference; Small interfering RNA 